\title{
The dCache Chemoreceptor TIpA of Helicobacter pylori Binds Multiple Attractant and Antagonistic Ligands via Distinct Sites
}

\author{
Kevin S. Johnson, ${ }^{a}$ (D) Bassam A. Elgamoudi, ${ }^{b}$ Freda E.-C. Jen, ${ }^{b}$ Christopher J. Day, ${ }^{b}$ Emily Goers Sweeney, ${ }^{c}$ Megan L. Pryce, ${ }^{a}$ \\ Karen Guillemin, ${ }^{\mathbf{c}}$ Thomas Haselhorst, ${ }^{\mathbf{b}}$ (1) Victoria Korolik, ${ }^{\mathbf{b}}$ (1) Karen M. Ottemann ${ }^{\mathbf{a}}$
}

\author{
aDepartment of Microbiology and Environmental Toxicology, University of California, Santa Cruz, California, USA \\ bInstitute for Glycomics, Griffith University, Gold Coast Campus, Southport, QLD, Australia \\ Institute of Molecular Biology, University of Oregon, Eugene, Oregon, USA
}

Kevin S. Johnson and Bassam A. Elgamoudi contributed equally to this work. Author order was decided based on project leadership and writing roles.

ABSTRACT The Helicobacter pylori chemoreceptor TIpA plays a role in dampening host inflammation during chronic stomach colonization. TlpA has a periplasmic dCache_1 domain, a structure that is capable of sensing many ligands; however, the only characterized TIpA signals are arginine, bicarbonate, and acid. To increase our understanding of TIpA's sensing profile, we screened for diverse TlpA ligands using ligand binding arrays. TIpA bound seven ligands with affinities in the low- to middle-micromolar ranges. Three of these ligands, arginine, fumarate, and cysteine, were TlpA-dependent chemoattractants, while the others elicited no response. Molecular docking experiments, site-directed point mutants, and competition surface plasmon resonance binding assays suggested that TlpA binds ligands via both the membranedistal and -proximal dCache_1 binding pockets. Surprisingly, one of the nonactive ligands, glucosamine, acted as a chemotaxis antagonist, preventing the chemotaxis response to chemoattractant ligands, and acted to block the binding of ligands irrespective of whether they bound the membrane-distal or -proximal dCache_1 subdomains. In total, these results suggest that TIpA senses multiple attractant ligands as well as antagonist ones, an emerging theme in chemotaxis systems.

IMPORTANCE Numerous chemotactic bacterial pathogens depend on the ability to sense a diverse array of signals through chemoreceptors to achieve successful colonization and virulence within their host. The signals sensed by chemoreceptors, however, are not always fully understood. This is the case for TlpA, a dCache_1 chemoreceptor of $H$. pylori that enables the bacterium to induce less inflammation during chronic infections. H. pylori causes a significant global disease burden, which is driven by the development of gastric inflammation. Accordingly, it is essential to understand the processes by which $H$. pylori modulates host inflammation. This work uncovers the signals that TIpA can sense and highlights the underappreciated ability to regulate chemotactic responses by antagonistic chemoreceptor ligands, which is an emerging theme among other chemotactic systems.

KEYWORDS Helicobacter pylori, chemotaxis, ligand discovery, dCache, signal transduction, chemoreceptor, inflammation, receptor-ligand interaction

hemotaxis is a vital host colonization strategy used by many pathogens, including Helicobacter pylori, Campylobacter jejuni, Borrelia burgdorferi, Pseudomonas aeruginosa, Vibrio cholerae, and Salmonella enterica. How chemotaxis benefits bacteria, however, varies. Pathogens have been found to use chemotaxis to access growth-promoting nutrients, locate signaling molecules that regulate virulence gene expression, spread throughout tissues and into specific niches, and affect host interactions that
Citation Johnson KS, Elgamoudi BA, Jen FE-C Day CJ, Sweeney EG, Pryce ML, Guillemin K, Haselhorst T, Korolik V, Ottemann KM. 2021 The dCache chemoreceptor TIpA of Helicobacter pylori binds multiple attractant and antagonistic ligands via distinct sites. mBio 12:e01819-21. https://doi.org/10.1128/mBio $.01819-21$

Editor Kelly T. Hughes, University of Utah Copyright $\odot 2021$ Johnson et al. This is an open-access article distributed under the terms of the Creative Commons Attribution 4.0 International license.

Address correspondence to Victoria Korolik, v.korolik@griffith.edu.au, or Karen M. Ottemann, ottemann@ucsc.edu.

This article is a direct contribution from Karen M. Ottemann, a Fellow of the American Academy of Microbiology, who arranged for and secured reviews by Gladys Alexandre, University of Tennessee at Knoxville, and Judith Armitage, University of Oxford.

Received 24 June 2021

Accepted 28 June 202

Published 3 August 202 
control inflammation (1). Chemotaxis signaling systems are highly conserved, and their widespread presence in pathogens underscores the importance of understanding their roles in colonization $(1,2)$.

One pathogen that requires chemotaxis for multiple infection aspects is $H$. pylori. This Gram-negative bacterium chronically colonizes the stomach of nearly half of the world's population and $\sim 35 \%$ of individuals in the United States (3). Stomach colonization results in chronic inflammation, and a subset of individuals develop ulcers and gastric cancer $(4,5)$. H. pylori presents a significant disease burden, with $\sim 700,000$ deaths from gastric cancer yearly (6). While many people are infected, the degree of host inflammation varies, which ultimately drives disease severity (7-9). We understand some $H$. pylori properties that dictate inflammation severity, such as the Cag pathogenicity island $(10,11)$. Still, the full compendium of $H$. pylori properties that modulate this host response is not yet understood.

H. pylori chemotaxis has been linked to host inflammation (12-15). Specifically, mutants missing key chemotaxis signal transduction proteins trigger less host inflammation despite achieving normal colonization levels, while mutants missing either the chemoreceptor TIpA or TlpB cause elevated inflammation (13-15). Chemoreceptors head the chemotaxis signal transduction system and dictate which signals a bacterium responds to. The loss of individual chemoreceptors within a system alters a bacterium's sensing profile but does not cause a complete loss of chemotactic ability, presumably biasing the bacterium toward signals sensed by the remaining chemoreceptors.

H. pylori possesses four chemoreceptors: TlpA, TlpB, TlpC, and TlpD. Each of these plays nonidentical roles in infection. TIpA, $-C$, and $-D$ are required for colonization, while TIpA and -B are required for inflammation control (15-17). In this work, we focus on TIpA, which plays multiple roles in promoting early but not late colonization and dampening later inflammation. At early times, 2 weeks postinfection, $H$. pylori $\Delta t / p A$ displays a modest colonization defect compared to the wild type (WT) as the sole infecting strain, a deficiency exacerbated by WT coinfection (15-17). However, during the chronic stage of infection after 6 months, $H$. pylori $\Delta$ tlpA bacteria colonize to normal levels but induce significantly more histologically evident inflammation than the WT (15).

TlpA is a transmembrane chemoreceptor with a periplasmic double-Cache (dCache_1) ligand binding domain (LBD) $(18,19)$. Cache domains are ubiquitous extracellular sensing domains found in both eukaryotes and prokaryotes, where they are the most common extracellular sensing domains (18). Cache domains bind a wide variety of small molecules but mostly amino acids, modified amino acids, and carboxylic acids (18). Many Cache domains have been found to bind multiple ligands (18). dCache_1 domains have two Cache subdomains, a membrane-distal and -proximal subdomain, each of which can bind ligands, although most commonly, the ligands are bound in the membrane-distal domain $(18,20,21)$. TlpA has some identified chemotaxis-active ligands, including arginine and sodium bicarbonate $(22,23)$. Additionally, TlpA has been shown to play a subtle role in sensing acidic pH but to a much lesser extent than TlpB or TIpD (24). Whether TlpA senses additional ligands or how any of these ligands are bound, however, is unknown.

Given the sensing potential of dCache_1 chemoreceptors, we hypothesized that TIpA would be capable of sensing ligands beyond those previously reported. Knowing a full set of ligands is critical for interpreting the TlpA-associated phenotypes. In this study, we identified new TIpA ligands and characterized their binding and ability to induce a chemotactic response. Ligand binding arrays were used to screen a broad set of ligands for binding to TIpA, resulting in the identification and verification of seven TIpA-specific ligands. The use of a temporal chemotaxis assay enabled us to determine that three ligands, arginine, fumarate, and cysteine, acted as TIpA-sensed chemoattractants, while the other ligands elicited no response. Molecular modeling experiments, assessment of TIpA point mutants, and surface plasmon resonance (SPR) competition assays suggested that TlpA ligands interact with two distinct sites. Furthermore, one of the high-affinity nonchemotactic TlpA ligands, glucosamine, blocked attractant 
TABLE 1 TIpA $A_{L B D}$ ligand binding analysis from the ligand binding array screen and surface plasmon resonance ${ }^{a}$

\begin{tabular}{lll}
\hline Ligand & Array result & Mean binding affinity $(\mu \mathrm{M}) \pm \mathrm{SD}$ \\
\hline Arginine & + & $2 \pm 0.11$ \\
Cysteine & $+/-$ & $4.7 \pm 0.3$ \\
Fumarate & + & $10 \pm 1.53$ \\
Glucosamine & + & $10.5 \pm 2.8$ \\
Malic acid & $+/-$ & $46 \pm 17$ \\
Thiamine & $+/-$ & $60 \pm 0.6$ \\
$\alpha$-Ketoglutarate & + & $224 \pm 11.2$ \\
\hline
\end{tabular}

a Data represent the mean values \pm SD from three independent experiments $(n=3)$. For the ligand binding array, results are reported as + for positive binding, $+/-$ for intermediate binding, and - for no binding. Binding affinity (micromolar) was determined by SPR.

responses to, and binding of, chemoattractant ligands, thus acting as an antagonist. Overall, our findings suggest that TIpA responds to several key $H$. pylori nutrients using both dCache_1 subdomains, with some acting as agonists and some acting as antagonists for a chemotaxis attractant response.

\section{RESULTS}

TIpA interacts directly with multiple ligands. TIpA's $L B D\left(T \mid P A_{L B D}\right)$ interactions with potential ligands were assessed using small-molecule arrays containing amino acids, organic acids, salts, and glycans (see Table $\mathrm{S} 1$ in the supplemental material). TlpA $\mathrm{ABD}_{\text {, }}$ bound seven small molecules: arginine, cysteine, fumarate, glucosamine, malic acid, thiamine, and $\alpha$-ketoglutarate (Table 1 and Fig. S1). Glycan arrays containing both simple and complex glycans were also interrogated, but no binding was detected.

We next determined $T \mid P A_{L B D}$ ligand binding affinities by surface plasmon resonance (SPR). Arginine, cysteine, fumarate, and glucosamine all exhibited high-affinity binding (dissociation constant $\left[K_{d}\right]$ of $<10 \mu \mathrm{M}$ ), while malic acid, thiamine, and $\alpha$-ketoglutarate showed lower affinities $\left(>45 \mu \mathrm{M}\right.$ ) (Table 1). Overall, these data suggest that the TIpA $\mathrm{L}_{\mathrm{LBD}}$ can interact directly with these seven ligands, with affinities ranging from 2 to $224 \mu \mathrm{M}$.

Some TIpA ligands act as chemoattractants, while others elicit no response. H. pylori chemotactic responses toward the seven putative TlpA-dependent ligands were examined by a live-cell video microscopy assay that measures the temporal chemotaxis response to test ligands. In this assay, attractants elicited fewer direction changes, and repellents elicited more direction changes, compared to basal levels (20, 25-30). Several TIpA ligands were acidic in solution, leading to the appearance of significant TlpA-independent chemorepellent responses; these were cysteine, thiamine, malic acid, and $\alpha$-ketoglutarate (Fig. S2A to C). Acidic conditions are sensed by chemoreceptors other than TIpA $(24,29,31)$ and potentially mask chemotactic responses to the ligands being tested. Accordingly, the $\mathrm{pH}$ of the ligand stocks for cysteine, thiamine, malic acid, and $\alpha$-ketoglutarate was neutralized using $\mathrm{NaOH}$ to match the $\mathrm{pH}$ of the water used in the mock-treated control. This treatment eliminated the confounding effect of medium acidification when assessing chemotactic responses (Fig. S2C). After incorporating these adjustments, we found that the addition of arginine, fumarate, or cysteine resulted in fewer direction changes for WT H. pylori (Fig. 1A to C), suggesting that these compounds were attractants. The highest concentration tested, $10 \mathrm{mM}$, induced the most significant and robust attractant responses for each ligand (arginine, $P<0.01$; fumarate, $P<0.01$; cysteine, $P<0.001$ ) (Fig. $1 \mathrm{~A}$ to $C$ ). Responses to 1 and $0.1 \mathrm{mM}$ ligands were apparent but not significant compared to the untreated control. Glucosamine, thiamine, malic acid, and $\alpha$-ketoglutarate induced no significant direction changes at any concentration tested (Fig. 1D and E), suggesting that they do not act as attractants or repellents.

To determine if chemoattractant responses toward arginine, fumarate, and cysteine were TIpA dependent, the same tracking experiments were repeated with a mutant lacking $t / p A(\Delta t \mid p A)$. The $\triangle t / p A$ mutant retained general chemotactic ability, producing significant attractant and repellent responses to the controls dipyridyl and $\mathrm{HCl}$, 
A.

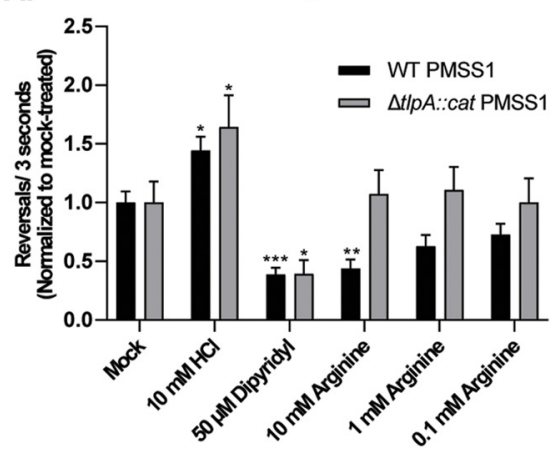

B.

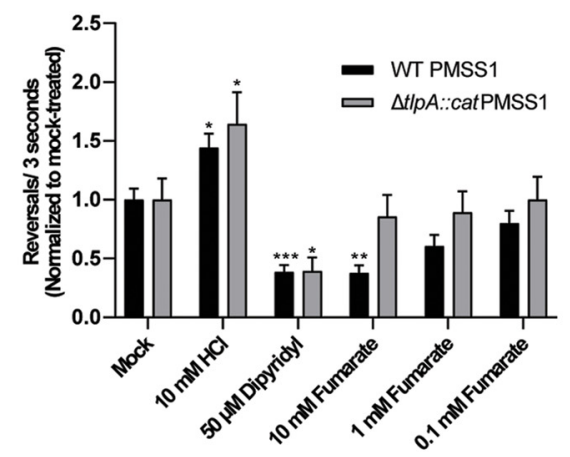

c.

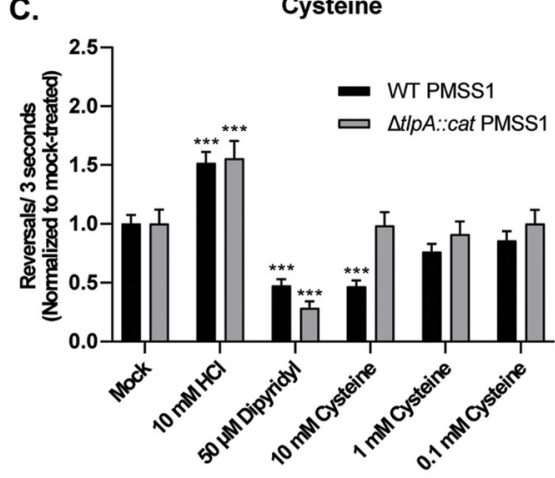

D.

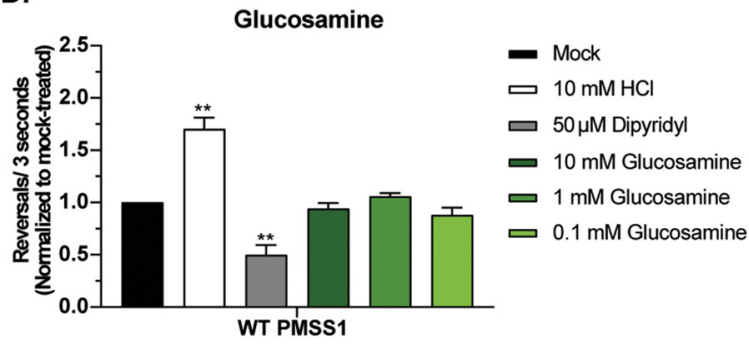

E.

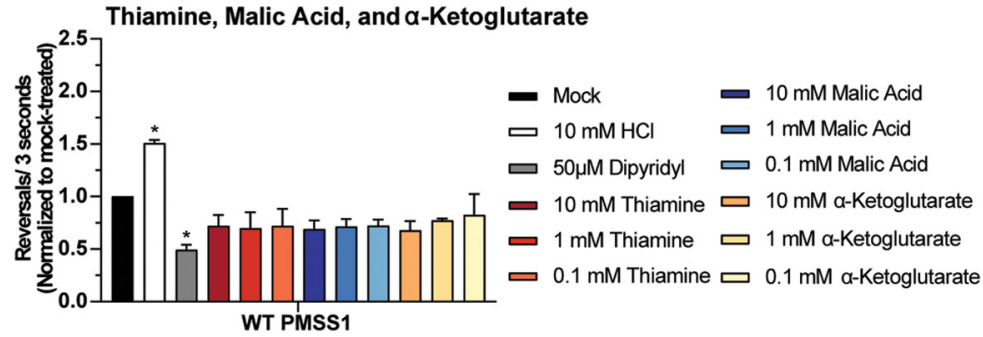

FIG $1 \mathrm{H}$. pylori responds to arginine, fumarate, and cysteine as TlpA-dependent chemoattractants in the temporal chemotaxis assay. Cultures of $H$. pylori PMSS1 WT and $\triangle$ t/pA strains were grown overnight, back-diluted, and then incubated until an $\mathrm{OD}_{600}$ of 0.12 to 0.15 was reached. Cultures were treated with water (Mock) or various concentrations of compounds, as indicated. For panels $\mathrm{C}$ and $\mathrm{E}$, the $\mathrm{pH}$ of cysteine, thiamine, malic acid, and $\alpha$-ketoglutarate stocks was adjusted using $\mathrm{NaOH}$ to match the $\mathrm{pH}$ of the water used for the untreated control. The cells were immediately filmed, and direction changes were counted over a 3-s swimming period in at least 100 cells per treatment from 3 biological replicates. Data are normalized to the values for the untreated control for each strain, as described in Materials and Methods. Error bars represent the standard errors of the means. ${ }^{*}, P<0.05 ;{ }^{* *}, P<0.01 ;{ }^{* * *}$, $P<0.001$ (comparisons to the untreated control per strain using two-way ANOVA with Dunnett's multiple-comparison test).

respectively, but failed to exhibit a chemotactic response to arginine, fumarate, or cysteine (Fig. 1A to C). These results suggest that the high-affinity ligands arginine, fumarate, and cysteine are TIpA-dependent chemoattractants.

TIpA has at least two ligand interaction sites. To gain insight into how TIPA binds ligands, a blind docking modeling experiment was carried out using AutoDock Vina $(32,33)$. We focused on two high-affinity ligands, arginine and fumarate. We found that these two ligands occupied two main sites, referred to as clusters (Table S2), which map to the membrane-distal and -proximal dCache_1 binding pockets (Fig. 2A and D). Arginine was placed mostly in cluster D (55\%) (Table S3), which is located in the membrane-distal dCache_1 domain. Arg153 dominated this binding interaction, with stabilization from Tyr151 (Fig. 2B and C). Fumarate, in contrast, was placed mostly in cluster A (45\%) (Table S3) in the membrane-proximal dCache_1 domain, with Phe203 being the most crucial residue required for interaction with fumarate (Fig. $2 \mathrm{E}$ and F). The docking experiment further revealed that although fumarate and arginine are likely to have two distinct preferred binding sites, they both can bind to their reciprocal sites. For example, $25 \%$ of the models had arginine found in fumarate's preferred cluster A (Table S3). Overall, these analyses suggest that arginine is more likely to bind the membrane-distal dCache_1 domain, while fumarate is more likely to bind the membrane-proximal dCache_ 1 domain, but binding to the other binding pockets is also possible.

To further study TlpA-ligand interactions, we generated TIpA $\mathrm{LBD}_{\mathrm{LD}}$ point mutants at residues in the membrane-distal (D165A and M183A) or membrane-proximal (Y228A, Y252A, and D254A) binding pockets and determined the binding affinity of the resultant proteins for all ligands (Table 2 and Fig. 3A). Mutation of either membrane-distal residue resulted in an $\sim 10$-fold decrease in the binding affinity for arginine, cysteine, fumarate, and glucosamine (Fig. $3 \mathrm{~B}$ and Table 2). Mutation of the membrane-proximal residue $T \mid p A_{Y 228 A}$ also led to a decrease in the binding affinity for arginine, cysteine, 
A.

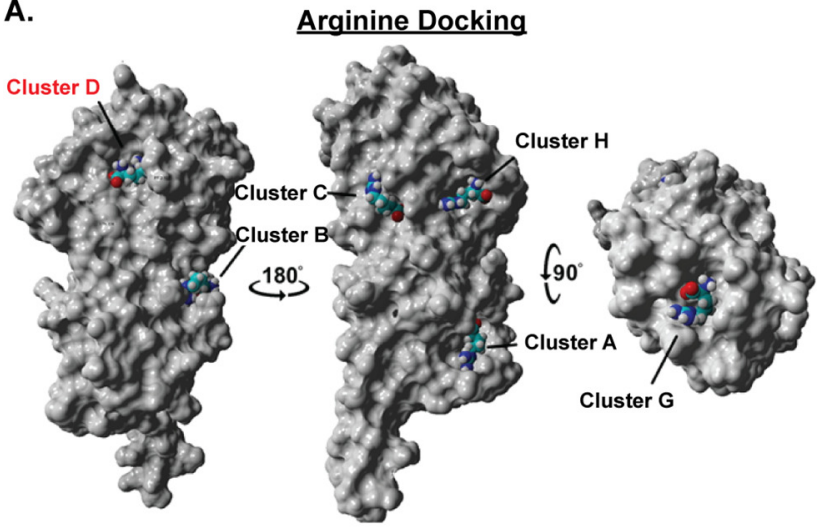

B.

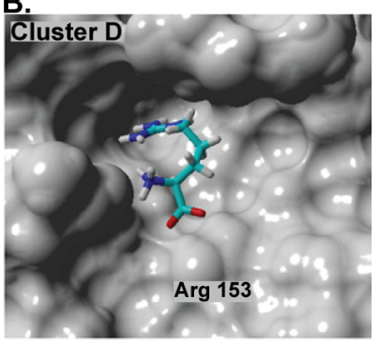

c.

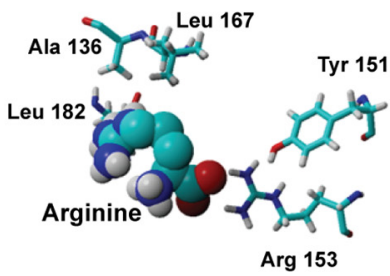

D.

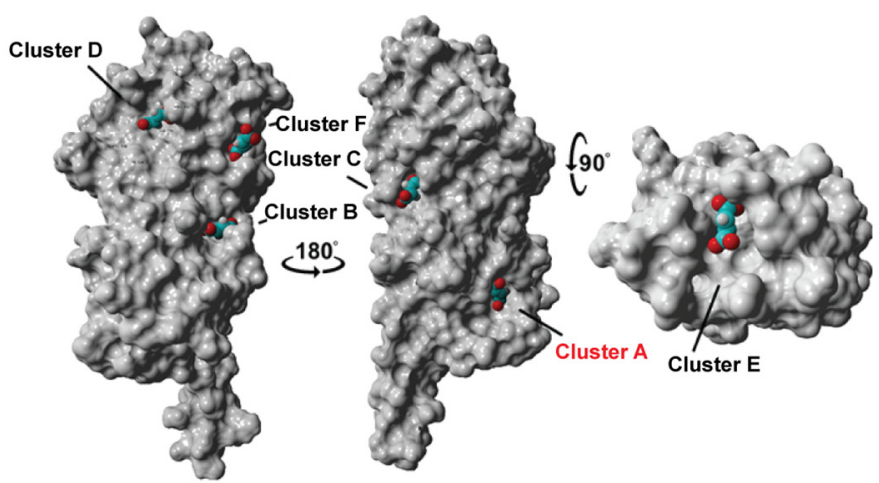

E.

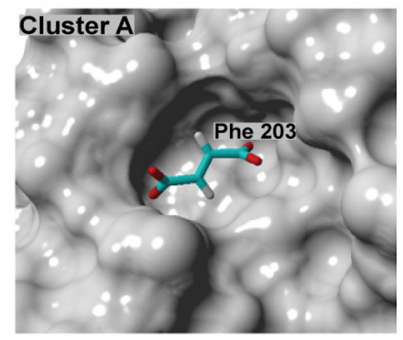

F.

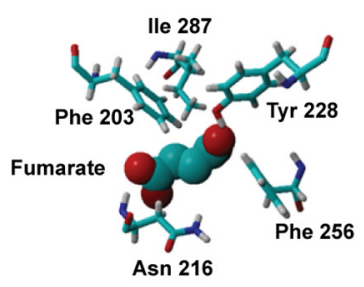

FIG 2 Docking analysis of TIPA and arginine or fumarate identifies several clusters that are occupied by these ligands. (A and D) Docking analysis shows several clusters occupied by arginine $(A)$ and fumarate $(D)$ on the surface of a space-filling version of Tlp $A_{L B D}$. Clusters $C$ and $G$ are biologically irrelevant due to the homodimer formation of TlpA, and clusters B and $\mathrm{H}$ are poorly populated (see Table S3 in the supplemental material). (B and C) View of the energetically preferred bound conformation of arginine in cluster $D$, the predicted membrane-distal dCache pocket, with key interacting amino acids shown. (E and F) View of the energetically preferred bound conformation of fumarate in cluster A, the membrane-proximal dCache pocket, with key interacting amino acids shown.

and fumarate, but it was only 4-fold. The other mutation in the membrane-proximal site $\left(\mathrm{TlpA}_{\mathrm{D} 254 \mathrm{~A}}\right)$ affected only fumarate binding (Fig. $3 \mathrm{~B}$ and Table 2). No proximal pocket residues affected the binding affinity of glucosamine. Membrane-distal and -proximal mutations resulted in a modest $\sim 3$ - to 4-fold increase in the binding affinity for malic acid and thiamine compared to the WT control. Additionally, no appreciable change in the binding affinity for $\alpha$-ketoglutarate was observed for any point mutant. Overall, TlpA binding interactions by the high-affinity ligands arginine, cysteine, fumarate, and glucosamine are most disrupted by mutations in the membrane-distal dCache_1 domain, but mutations in the membrane-proximal dCache_1 also significantly impair fumarate and, to a lesser extent, arginine and cysteine binding, consistent with the predictions from the docking analysis.

TIPA $_{\text {LBD }}$ binds arginine and fumarate through distinct binding sites. The abovedescribed data suggest that $\mathrm{TIPA}_{\mathrm{LBD}}$ can bind ligands in both dCache_1 subdomain binding pockets. To further analyze the possibility of two distinct binding sites for chemotaxis-active ligands in $T_{1 p} A_{L B D}$, we employed a competition SPR (A-B-A) binding

TABLE 2 Binding affinities of TlpA $A_{L B D}$ and TlpA $A_{L B D}$ membrane-distal and -proximal dCache mutants for TlpA ligands ${ }^{a}$

\begin{tabular}{lllllll}
\hline \multirow{2}{*}{ Ligand } & \multicolumn{7}{l}{ Mean binding affinity $(\mu \mathrm{M}) \pm \mathrm{SD}$} \\
\cline { 2 - 7 } & $\mathrm{TlpA}_{\mathrm{WT}}$ & $\mathrm{TlpA}_{\mathrm{D} 165 \mathrm{~A}}$ & $\mathrm{TlpA}_{\mathrm{M} 183 \mathrm{~A}}$ & $\mathrm{TlpA}_{\mathrm{Y} 228 \mathrm{~A}}$ & $\mathrm{TlpA}_{\mathrm{Y} 252 \mathrm{~A}}$ & $\mathrm{TlpA}_{\mathrm{D} 254 \mathrm{~A}}$ \\
\hline Arginine & $2 \pm 0.11$ & $12.4 \pm 0.84$ & $22.3 \pm 5.9$ & $8.3 \pm 1.6$ & $4.96 \pm 1.8$ & $3.71 \pm 0.73$ \\
Cysteine & $4.7 \pm 0.3$ & $41.9 \pm 0.4$ & $36.1 \pm 15.7$ & $15.7 \pm 6.6$ & $5.5 \pm 0.8$ & $4.58 \pm 1.5$ \\
Fumarate & $10 \pm 1.5$ & $100.5 \pm 38.9$ & $106.1 \pm 36.6$ & $46.6 \pm 3.9$ & $8.5 \pm 1.7$ & $44 \pm 8.1$ \\
Glucosamine & $10.5 \pm 2.8$ & $96 \pm 24.3$ & $94.6 \pm 57.3$ & $17.1 \pm 70$ & $2.96 \pm 0.7$ & $9.84 \pm 3.7$ \\
Malic acid & $46 \pm 17$ & $11.9 \pm 4.3$ & $33.6 \pm 6.2$ & $28.6 \pm 9.4$ & $14.2 \pm 6.2$ & $29.3 \pm 2$ \\
Thiamine & $60 \pm 0.6$ & $43.9 \pm 14.1$ & $18.1 \pm 1.2$ & $29.5 \pm 12.3$ & $35 \pm 7.3$ & $39.3 \pm 4.3$ \\
$\alpha$-Ketoglutarate & $224 \pm 11.2$ & $124 \pm 7.1$ & $256.2 \pm 27.6$ & $295.4 \pm 27.7$ & $289 \pm 22.6$ & $321 \pm 21.6$ \\
\hline
\end{tabular}

${ }^{a}$ Data represent the mean values \pm SD from three independent experiments $(n=3)$. 
A.

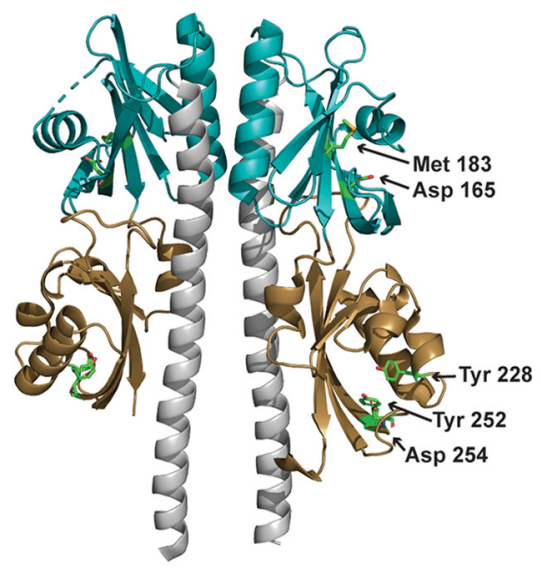

B.

\section{TIpA Point Mutants}

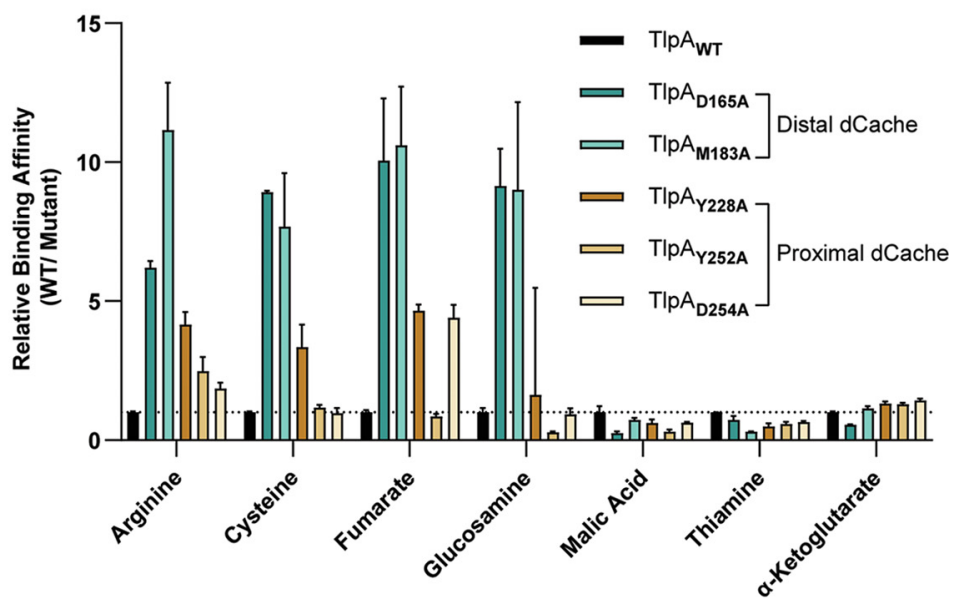

FIG 3 TIPA $_{L B D}$ binds chemotaxis-active ligands through the membrane-distal or -proximal dCache subdomains. (A) Ribbon diagram of $\mathrm{TIPA}_{\perp B D}$ as a homodimer. The membrane-distal and membrane-proximal dCache domains are shown in teal and gold, respectively.

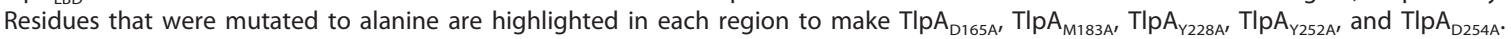
(B) Relative binding affinities of each ligand for the WT and TIpA example, mutation of D165 to A resulted in an $\sim 6$-fold decrease in the arginine binding affinity compared to WT binding. Data represent the mean values and standard errors of the means from three independent experiments $(n=3)$.

assay focused on arginine and fumarate because they were both chemoattractants and predicted to bind different sites preferentially. In this assay, the competition for binding to $\mathrm{TIPA}_{\mathrm{LBD}}$ between arginine and fumarate is assessed by adding the ligands sequentially and monitoring whether the SPR signal changes upon the addition of the second ligand. The two ligands' binding status can be classified as either independent, shared, or preferential shared sites. For independent sites, ligand A saturates all its binding sites, and ligand $B$ then binds to its independent site; this mode produces additive effects on the SPR signal. Shared sites, in contrast, do not produce additive/cumulative effects; i.e., ligand A binds its site and then blocks ligand B from the same site. Finally, it is also possible to have preferential shared sites where ligands share the same binding site, but the protein binds to one ligand preferentially when in equilibrium.

We first saturated $\mathrm{TlPA}_{\mathrm{LBD}}$ with arginine and then added fumarate. In this case, an increased response (additive effect) was observed, compared to the theoretical value (Fig. 4). This outcome suggests that fumarate and arginine bind to independent sites. Conversely, when TIPA $A_{L B D}$ was saturated with fumarate, arginine did not produce an additional response, compared to the theoretical value (Fig. 4). This result suggests that fumarate prevented arginine binding because either arginine competed with fumarate at the same site(s) or fumarate caused an allosteric effect that prevents arginine binding, a common occurrence in sensory proteins (34). Overall, the docking and competitive SPR data support the hypothesis that there are two binding sites with possible cooperative interactions or overlap between them.

The docking analysis and competition SPR assay suggested that arginine and fumarate bind to distinct $\mathrm{TIPA}_{\mathrm{LBD}}$ sites; therefore, we sought to further characterize TlpA-ligand interactions using saturation transfer difference (STD) nuclear magnetic resonance (NMR) spectroscopy, which can measure protein-ligand interactions and ascertain which part of a ligand interacts with the receptor protein $(35,36)$. When $\mathrm{TIPA}_{\mathrm{LBD}}$ bound fumarate, a significant STD NMR signal was detected, consistent with the two ethylene protons interacting with the protein (Fig. 5A). Similarly, when $\operatorname{TlpA}_{L B D}$ bound arginine, significant STD NMR signals were observed (Fig. 5B). On arginine, the relative STD NMR effects showed that the $\mathrm{H}-3$ and $\mathrm{H}-4$ protons on the side chain received the largest saturation transfer from the protein protons, indicating that arginine interacts with $\mathrm{TlpA}_{\mathrm{LBD}}$ around its middle carbon side chain region (Fig. $5 \mathrm{~B}$ ). These results therefore provide additional confirmation 
Competitive SPR: Argininevs. Fumarate

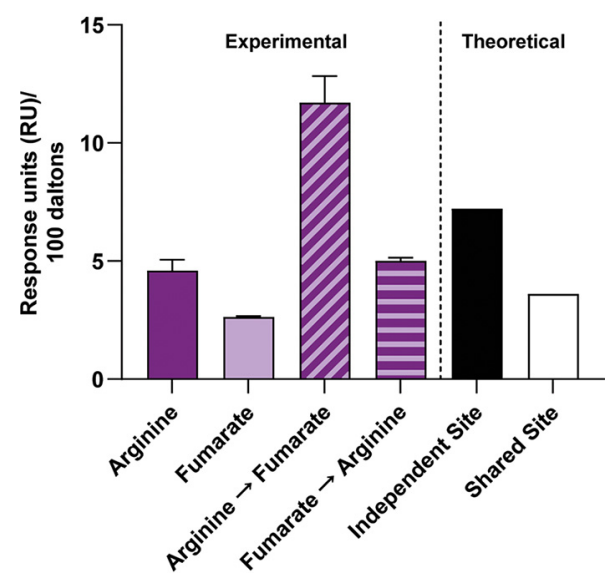

FIG 4 SPR competition analysis indicates the presence of two distinct binding sites for TIpA ligands. SPR competition analyses of binding by arginine and fumarate to WT TIPA $A_{L B D}$ were performed. Compounds were used at concentrations 10 -fold higher than their respective $K_{d}$ values. Arginine, response to arginine only; Fumarate, response to fumarate only; Arginine $\rightarrow$ Fumarate, fumarate response after saturation with arginine; Fumarate $\rightarrow$ Arginine, arginine response after saturation with fumarate. The theoretical values are responses units based on mathematical theory: independent site is the sum of individual responses, and shared site is the sum of individual responses divided by the number of individual responses. All response data were normalized to a molecular weight of $100 \mathrm{Da}$ for each analyte, allowing direct comparison of responses.

that TlpA interacts with fumarate and arginine, mostly along the carbon chain backbones in each ligand.

A non-chemotaxis-active TIpA ligand can antagonize chemoattractant responses. It was surprising to find a high-affinity direct binding ligand, glucosamine, that bound to the membrane-distal dCache_1 subdomain (Fig. 3) and did not elicit a chemotaxis response (Fig. 1D). Previous reports on ligand interactions with chemoreceptors in Escherichia coli and P. aeruginosa suggested that some ligands bind chemoreceptors as antagonists, blocking normal chemotactic responses toward chemotaxis-active ligands $(37,38)$. Consequently, we tested whether glucosamine could block the binding of the chemotaxis-active TIpA ligands arginine and fumarate using a competitive SPR assay. Of note, the other nonchemoactive TlpA ligands, malic acid, thiamine, and $\alpha$-ketoglutarate, were not affected by either membrane-proximal or -distal dCache_1 domain point mutants (Fig. 3); therefore, we hypothesized that they would be unable to affect the

A. $\mathrm{H}^{1}, \mathrm{H}^{2}$

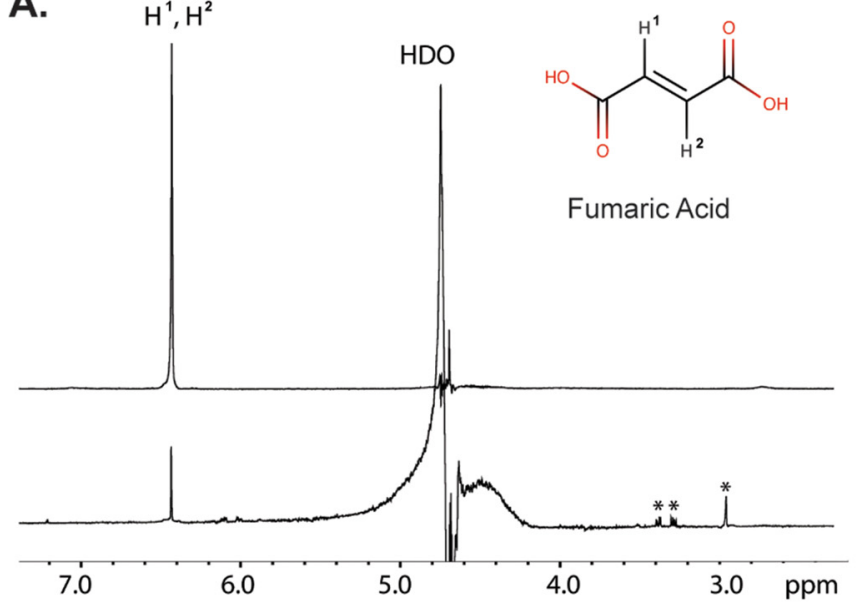

B.

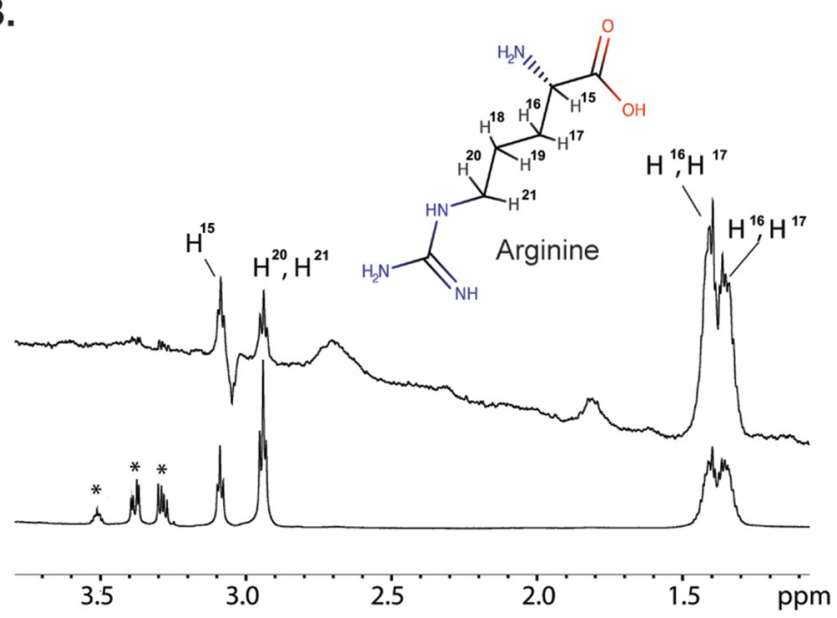

FIG 5 STD NMR analysis supports that TIPA $A_{L B D}$ binds fumarate and arginine. ${ }^{1} \mathrm{H}$ NMR spectra are shown at the bottom for fumarate (A) and arginine (B). The STD NMR spectra are shown at the top, acquired at $600 \mathrm{MHz}$ at $289 \mathrm{~K}$, with an on-resonance of -1 ppm, an off-resonance of 33 ppm, and a total saturation time of $2 \mathrm{~s}$. 
A.

Competitive SPR: Arginine vs. Glucosamine

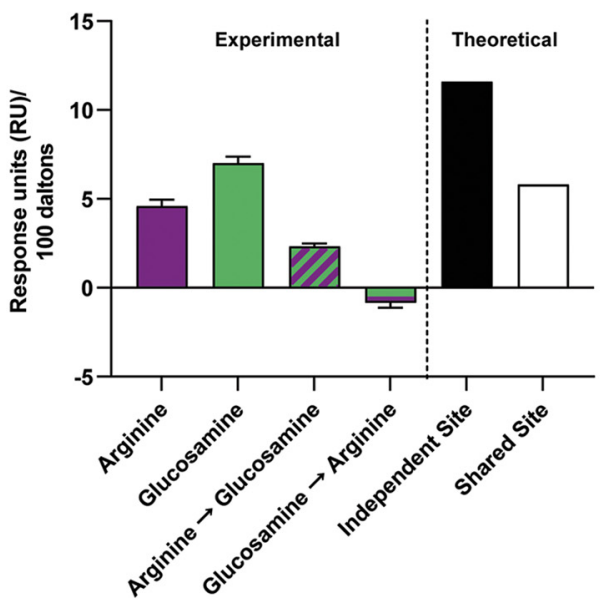

B.

Competitive SPR: Fumarate vs. Glucosamine

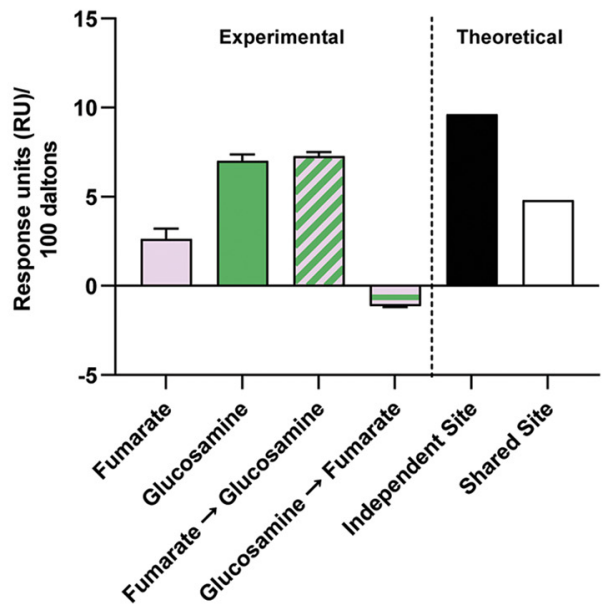

FIG 6 SPR competition analysis demonstrates that glucosamine blocks the binding of TlpA chemoattractants. Data from SPR competition analysis of binding of arginine, fumarate, and glucosamine to WT TIpA $\mathrm{LBD}_{\mathrm{LB}}$ are shown. Compounds were used at concentrations 10-fold higher than their respective $K_{d}$ values. Arginine, response to arginine only; Glucosamine, response to glucosamine only; Arginine $\rightarrow$ Glucosamine, response to glucosamine following saturation with arginine; Glucosamine $\rightarrow$ Arginine, response to arginine following saturation with glucosamine; Fumarate, response to fumarate only; Fumarate $\rightarrow$ Glucosamine, response to glucosamine following saturation with fumarate; Glucosamine $\rightarrow$ Fumarate, response to fumarate following saturation with glucosamine. The theoretical values are response unit values based on mathematical theory. All response data were normalized to a molecular weight of $100 \mathrm{Da}$ for each analyte, allowing direct comparison of responses.

binding of chemotaxis-active TIpA ligands, as they do not appear to bind through the same sites. The results showed that when glucosamine was added following saturation with arginine or fumarate, the response was not additive, suggesting that glucosamine competed with both arginine and fumarate (Fig. 6A and B). However, when arginine or fumarate was added to TIPA $_{L B D}$ following initial saturation with glucosamine, no additive response was observed (Fig. $6 \mathrm{~A}$ and $\mathrm{B}$ ). This result suggests that glucosamine can prevent the binding of both ligands to $T / p A_{L B D}$.

Consequently, we tested whether glucosamine affected $H$. pylori chemotaxis by developing a ligand competition tracking assay between non-chemotaxis-active and chemotaxis-active TIpA ligands. This assay is a modified version of our live-cell video microscopy assay where the addition of a chemotaxis-active ligand is followed by the addition of a non-chemotaxis-active ligand $10 \mathrm{~s}$ later and vice versa. Using this approach, we determined that glucosamine addition prevented the chemoattractant response to arginine and fumarate and severely blunted the response to cysteine (Fig. 7). This response was decreased regardless of whether glucosamine was added before or after the addition of the chemoattractant. In total, these results suggest that glucosamine blocks chemotaxis-active ligand binding and acts as a TlpA chemotaxis antagonist.

\section{DISCUSSION}

We report the identification of several ligands specific for the $H$. pylori dCache_1 chemoreceptor TlpA, including confirmation of previous reports that TlpA interacts with arginine $(22,23)$. Arginine, along with fumarate and cysteine, functioned as a TlpA-sensed chemotaxis attractant. Furthermore, these chemotaxis-active ligands appeared to interact with the membrane-distal and -proximal dCache_1 domains. Finally, we found that glucosamine acts as a chemotaxis antagonist, blocking TlpA binding and responses to multiple attractants.

TIpA can bind a broad set of ligands with diverse biological functions. TlpA bound a broad set of molecules ranging from the amino acid arginine, with a large, charged side chain; to the amino acid cysteine, with a smaller, polar side chain; 
A.

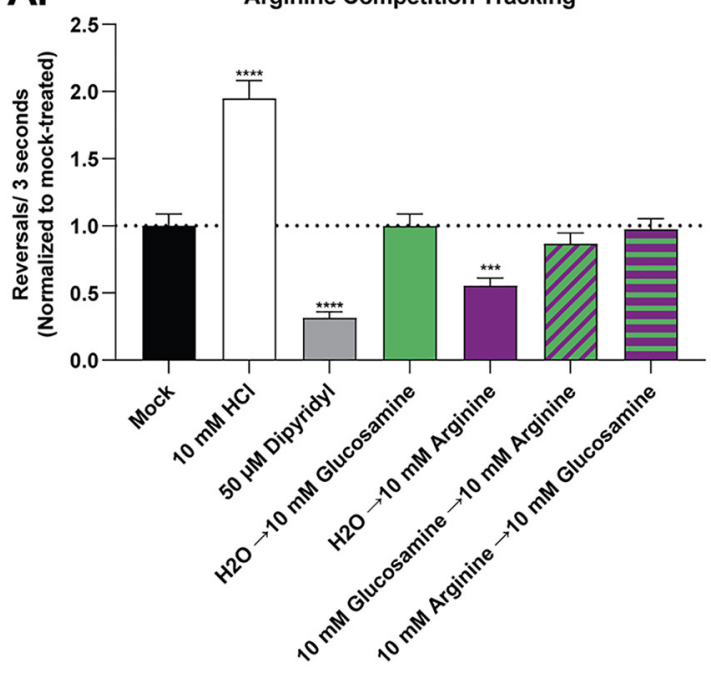

\section{C.}

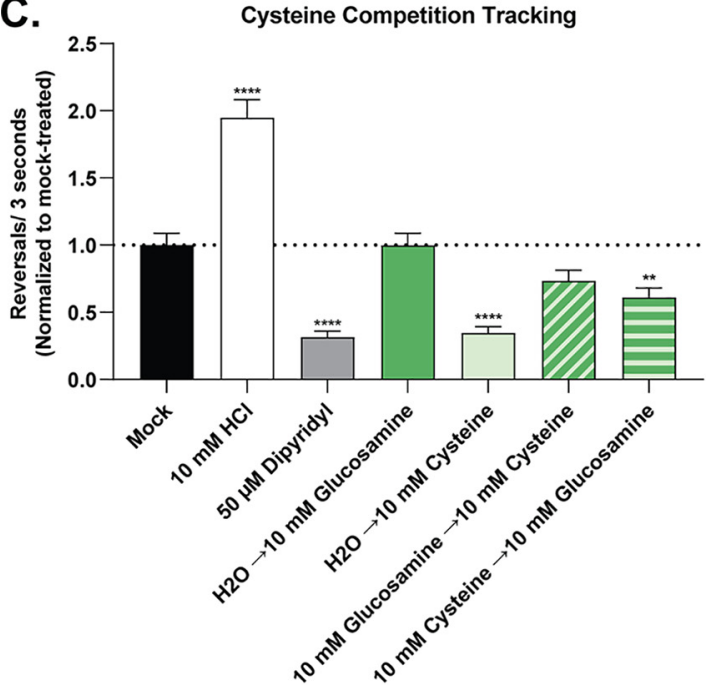

B.

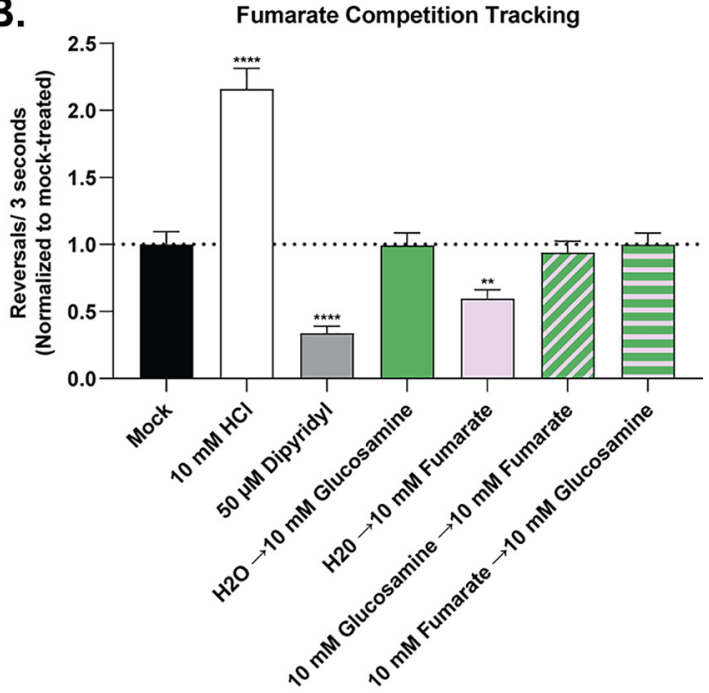

FIG 7 Ligand competition tracking experiment between chemoactive and nonchemoactive TlpA ligands. Cultures of the $H$. pylori PMSS1 WT were grown in BB10 overnight and then back-diluted as described in the legend of Fig. 1. Cultures were mock treated or treated with various concentrations of compounds as indicated. The $\mathrm{pH}$ of the cysteine stock was adjusted using $\mathrm{NaOH}$ to match the $\mathrm{pH}$ of the water used for the untreated control. The cells were immediately filmed, and direction changes were counted over a 3-s swimming period in at least 100 cells per treatment from 3 biological replicates. Repellents increase direction changes, as exemplified by the control repellent $\mathrm{HCl}$, while attractants decrease direction changes, as exemplified by the control attractant dipyridyl. Data are normalized to the values for the untreated control for each strain, as described in Materials and Methods. Error bars represent the standard errors of the means. ${ }^{*}, P<0.05 ;{ }^{* *}, P<0.01$; ${ }^{* *}, P<0.001$ (comparisons to the untreated control per strain using two-way ANOVA and Dunnett's multiple-comparison test).

organic acids, including fumarate, malic acid, and $\alpha$-ketoglutarate; the large vitamin thiamine; and the amino sugar glucosamine. Ultimately, our data suggest that only some of these ligands bind within the canonical dCache_1 binding pockets, including all those that affected chemotaxis. Thus, these results agree with previous reports showing that individual dCache chemoreceptors can sense diverse types of ligands $(39,40)$.

The three molecules that elicited a chemotaxis response, arginine, fumarate, and cysteine, have been shown to have important biological roles in $H$. pylori biology. Arginine is an essential amino acid for $H$. pylori under in vitro growth conditions (41), and $H$. pylori uses arginine to promote acid tolerance and dampen host immune responses (42-45). Fumarate is predicted to be an alternative terminal electron acceptor for growth under anaerobic respiration (46), and the associated enzyme, fumarate reductase, is essential for $H$. pylori colonization in vivo (47). Additionally, 
fumarate is highly depleted when $H$. pylori is cocultured with gastric organoids, consistent with the prediction that it is preferentially used in vivo (48). Finally, cysteine is an essential amino acid for some strains of $H$. pylori (41). These ligands have been shown to be present in the stomach during infection via metabolomics studies $(48,49)$; however, the exact concentration of these ligands is not known. Each chemotaxis-active TIpA ligand is important for critical cellular functions for $H$. pylori; therefore, the ability to sense these ligands is a likely survival-linked evolutionary adaptation.

TIpA appears to use both binding pockets. dCache_1 chemoreceptors contain two potential ligand binding pockets in each Cache subdomain. Previous work showed that dCache_1 chemoreceptors sense chemotaxis-active ligands through either subdomain but, as of yet, not both $(20,21)$. In contrast, our data suggest that TlpA might bind ligands in both Cache subdomains. Ligand binding locations, suggested by docking prediction analysis, placed fumarate within the membrane-proximal subdomain and arginine within the membrane-distal subdomain. These location assignments were further supported by TIPA $_{L B D}$ point mutants of residues within the predicted dCache_1 membrane-distal (D165A and M183A) and membrane-proximal (Y228A, Y252A, and D254A) subdomains. All membrane-distal subdomain mutations led to a decreased binding affinity for the chemotaxis-active ligands arginine, fumarate, and cysteine as well as the antagonist ligand glucosamine. These findings suggest that the membrane-distal site is important for chemotaxis signaling, as seen in other dCache_1 receptors $(18,21)$. It has been shown that individual dCache_1 receptors can bind aliphatic, small polar, and large positively charged amino acids through a single subdomain due to the malleable nature of dCache_1 receptors that can accommodate ligands of different sizes and charges (40). Thus, it is plausible that the membrane-distal subdomain could be able to accommodate these diverse ligands.

Our data also suggest that the membrane-proximal subdomain plays a role in TlpA ligand binding. Site-directed mutagenesis of the membrane-proximal subdomain, as well as the membrane-distal one, decreased fumarate binding. This outcome suggests that the point mutations either directly disrupted ligand binding or altered long-range interactions in the protein that influence ligand binding affinities. Additionally, our data showed that fumarate blocked arginine binding despite having similar binding affinities. Of note, arginine binding to the membrane-proximal domain was affected by

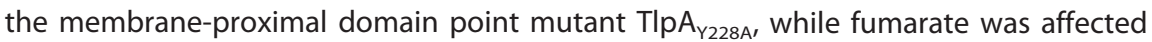
by both the TIpA $\mathrm{Y}_{222 \mathrm{~A}}$ and $\mathrm{TIpA}_{\mathrm{D} 254 \mathrm{~A}}$ membrane-proximal domain point mutants, which supports docking analysis predictions suggesting that fumarate preferentially binds to the membrane-proximal domain, while arginine preferentially binds to the membranedistal domain. Several models could account for these findings. One is that fumarate binds at the membrane-proximal site and creates an allosteric change that prevents ligand binding at the membrane-distal site. Alternatively, the amino acid changes in the proximal site could affect the ligand affinities at the distal site. Finally, a third possibility is that arginine binds at both sites, and proximal-site binding is affected by the proximal mutations. It will be interesting to dissect whether there are cooperative interactions, distinct site binding, or simultaneous site binding. Regardless, while cooperativity between subdomains in dCache_1 chemoreceptors has not yet been observed, it is well documented that four-helix-bundle types of chemoreceptors have negative cooperativity between their two binding sites (34). Furthermore, it is not yet known which site is required for chemotaxis. Overall, our data suggest that TIpA may use both dCache_1 subdomains to bind ligands.

TIpA chemotaxis responses can be antagonized. We were somewhat surprised to find a high-affinity-binding TlpA ligand, glucosamine, that did not elicit a chemotaxis response yet appeared to bind the membrane-distal dCache_1 subdomain. Indeed, we found that glucosamine occluded chemotaxis-active TIpA ligands from binding and inhibited the normal chemoattractant responses to arginine, fumarate, and cysteine. This response was observed regardless of whether glucosamine was added before or after the addition of the TIpA chemoattractants, suggesting that glucosamine may have a very high on-rate for binding TIpA compared to arginine, fumarate, or cysteine. 
Two studies have reported high-affinity chemoreceptor ligands that acted as antagonists by blocking chemotaxis-active ligand binding $(37,38)$, and Cache receptor antagonists have been reported for a histidine kinase (50). Martín-Mora and colleagues showed that the binding of the attractant malic acid to the $P$. aeruginosa sCache chemoreceptor PA2652 was inhibited by either citraconic acid or D,L-methylsuccinic acid, and subsequently, chemoattractant responses to malic acid were decreased (37). Another of these studies described finding diverse ligands for the $E$. coli fourhelix-bundle chemoreceptor Tar and reported that a high-affinity ligand, cis-1,2cyclohexane-dicarboxylic acid, also acted as an antagonist for aspartate chemotaxis. cis-1,2-Cyclohexane-dicarboxylic acid competed for aspartate binding and blocked intracellular kinase activity (38). We expect that there will be more discoveries of these types of chemomodulatory antagonists, or maybe even chemotaxis-enhancing ligands, because ligand discovery methods have changed. Specifically, previous efforts relied on chemotaxis assays, and so only chemotaxis-active ligands could be identified. In contrast, recent approaches look for direct ligand-receptor interactions at the molecular level, thus expanding our ability to identify the interacting partners $(21,38,51-53)$.

The function of chemoreceptor antagonists is not yet known in any system (37, 38). In the case of TIpA, it is possible to speculate that when confronted with abundant glucosamine, $H$. pylori benefits by not responding to arginine, fumarate, or cysteine. However, the role of glucosamine in $H$. pylori infection is unknown, although it has been shown to support the growth of some $\mathrm{H}$. pylori clinical isolates using phenotypic Biolog plates (54). One possibility is that antagonist ligands may function as a form of adaptation, as $H$. pylori lacks the classical adaptation proteins CheR and CheB (55), possibly in lieu of, or in augmentation to, other adaptation systems. Chemotaxis antagonists may be useful tools to modulate chemotaxis and affect bacterial pathogenesis. In the case of TIpA, blocking its function early in infection would decrease colonization; however, later attenuation of chemotactic responses might be predicted to enhance inflammation (15-17). Future work performing molecular dynamics experiments to understand how agonist and antagonist ligands interact with $\mathrm{TIpA}_{\mathrm{LBD}}$ will help us to understand the function of chemoreceptor antagonists.

One caveat of this study is that experimental analyses were carried out under two different conditions. The ligand binding work was all done with purified TIPA $A_{L B D}$, while the chemotaxis studies were done on full-length TIpA that was in the context of both a membrane and its interactions with other chemoreceptors. These two different situations may lead to varying outcomes. For example, it is not clear how ligand interactions would change in the context of chemosensory array-packed receptors, which is an area for future work.

Expanding the knowledge of TlpA ligands, and how TlpA interacts with these ligands, is essential for better understanding why TIpA enhances the in vivo fitness of $H$. pylori and alters inflammatory phenotypes driven by $H$. pylori. Future experiments manipulating the ability of $H$. pylori to sense specific TlpA ligands will be useful to understand whether all or a subset of TlpA ligands play a role in driving these in vivo phenotypes (15-17). Furthermore, this work provides another example $(37,38)$ of a chemotaxis system having antagonistic ligands, operating through a distinct type of chemoreceptor ligand binding domain. These results suggest an interesting possible mechanism for regulating responses to multiple chemotactic ligands in a nutrient-rich environment using agonist and antagonist ligands.

\section{MATERIALS AND METHODS}

TIpA construct design and protein purification. The periplasmic portion of TIpA $\left(T \mid p A_{L B D}\right)$, amino acids 28 to 299 , from Helicobacter pylori SS1 was cloned into a pBH4 expression vector ( $\mathrm{pBH} 4$ _TIpA $\mathrm{LBD}_{\mathrm{LD}}$ ) to generate an $\mathrm{N}$-terminal $6 \times$ His-tagged construct, with a tobacco etch virus (TEV) protease site, under the control of isopropyl- $\beta$-D-thiogalactopyranoside (IPTG) (19). Alanine point mutants at Asp165, Met183, Tyr228, Tyr252, and Tyr254 in $\mathrm{TIPA}_{\mathrm{LBD}}$ were generated via site-directed mutagenesis of pBH4_TlpA with primers listed in Table $\mathrm{S} 4$ in the supplemental material and confirmed via restriction digestion and 
sequencing (QuikChange; Stratagene). TIpA ${ }_{\perp B D}$ and all point mutants were purified as described previously by Sweeney et al. (19). Circular dichroism (CD) spectroscopy was used to confirm the correct folding of all proteins (Fig. S3) (56).

Ligand binding array. Small-molecule arrays were prepared and performed as previously described (57). Briefly, $1 \mu \mathrm{g}$ of purified TlpA $\mathrm{LBD}_{\text {in }}$ in phosphate-buffered saline (PBS) (pH 7.2) was incubated with a molar concentration ratio (4:2:1) of anti-His antibody (Cell Signaling), followed by incubation with a secondary antibody (Thermo Scientific) for signal amplification. The protein-antibody mix was added to an Arrayit Superepoxy III glass substrate array blocked with PBS (pH 7.2) with $1 \%$ bovine serum albumin. The glass substrate array was printed with quadruplicate spots of 148 different amino acids, salts of organic acids, and other small molecules (Table S1). Unbound protein was washed away with PBS with $0.05 \%$ Tween. The arrays were scanned by a ProScan array scanner at $488 / 520 \mathrm{~nm}$, and the results were analyzed by the ScanArray Express software program (PerkinElmer). Three biological replicates were performed, with a total of 12 data points for each glycan tested. Binding was classified as positive for a ligand if the relative fluorescence unit value was $>1$-fold above the mean background (defined as the average background of negative-control spots plus 3 standard deviations [SD]) and was statistically significant $(P<0.005$ by Student's $t$ test). Small-molecule array slide preparation and analysis were done according to MIRAGE guidelines (58) (outlined in Table S5).

Surface plasmon resonance measurements. Purified $\mathrm{TlpA}_{\mathrm{LBD}}$ was immobilized on a CM5 series $\mathrm{S}$ sensor chip, and binding affinities were tested using a Biacore S200 instrument (GE Healthcare) as described previously $(39,57,59)$. Briefly, proteins were captured using an amine-coupling kit (GE Healthcare), in which the carboxylmethyl dextran matrix of the sensor chip was activated by the injection of a mixture of $0.2 \mathrm{M}$ 1-ethyl-3-[(3-dimethylamino)propyl]-carbodiimide (EDC) and $0.05 \mathrm{M} \mathrm{N}$-hydroxysuccinimide (NHS), followed by the neutralization of the remaining unreacted NHS ester groups by the injection of $1 \mathrm{M}$ ethanolamine- $\mathrm{HCl}\left(\mathrm{pH}\right.$ 8.0). Purified $\mathrm{TlpA}_{\mathrm{LBD}}$ was diluted in $10 \mathrm{mM}$ sodium acetate buffer ( $\mathrm{pH} 4.5$ ) at a concentration of $100 \mu \mathrm{g} / \mathrm{ml}$ for immobilization to the chip. A total of 8,400 response units $(\mathrm{RU})$ of $\mathrm{TIPA}_{\mathrm{LBD}}$ were captured on flow cell 2 . As a negative control, flow cell 1 was a blank control undergoing the same treatment as the other flow paths, without the protein injection. This set enabled double-reference subtraction of the responses $(2-1,3-1$, and $4-1)$. The tested compounds were prepared as a stock concentration of 100 to $200 \mathrm{mM}$ in PBS. The compounds were then diluted between $1 \mathrm{nM}$ and $1 \mathrm{mM}$ in a series of 1:10 dilutions in PBS and run over the flow cells at a flow rate of $30 \mu \mathrm{l} / \mathrm{min}$. Between each sample testing, a series of buffer-only injections was run to enable double-blank subtraction for the sensorgram assessment. After the initial run, based on the results, the dilution series ranged from $0.195 \mu \mathrm{M}$ to $1 \mathrm{mM}$ in 1:4 dilutions in PBS. The samples were then run using single-cycle kinetic/affinity methods in triplicate for those compounds that showed submillimolar affinity after the initial binding screen. The data sets were analyzed using Biacore S200 evaluation software 2.0.2; sensorgrams were double-reference subtracted.

Bacterial strains and growth conditions. For all chemotaxis assays, $H$. pylori strain PMSS1 was used (9). Bacteria were grown in Brucella broth (BD BBL/Fisher) with 10\% heat-inactivated fetal bovine serum (FBS) (Life Technologies) (BB10), with shaking, at $37^{\circ} \mathrm{C}$ under microaerobic conditions of $5 \% \mathrm{O}_{2}, 10 \% \mathrm{CO}_{2}$, and $85 \% \mathrm{~N}_{2}$. The PMSS1 $\Delta$ t/pA mutant was created by the natural transformation of wild-type PMSS1 with $5 \mu \mathrm{g}$ of $\Delta$ tlpA::cat SS1 genomic DNA (16). Chloramphenicol-resistant mutants were selected using $10 \mu \mathrm{g} / \mathrm{ml}$ chloramphenicol on Columbia horse blood agar as previously described (16). Mutation of t/pA was confirmed by PCR amplification of genomic DNA from WT PMSS1, $\Delta$ t/pA::cat PMSS1, and $\Delta$ t/pA::cat SS1 using primers TIPA_SS1_5' (TTGTCTAAAGGTTTGAGTATC) and TIpA_SS1_3' (TTAAAACTGCTTTTATTCAC) (this study) (Fig. S4).

Chemotaxis assays. Swimming behavior assays were done with $H$. pylori PMSS1 strains grown in BB10 as described above. Cultures grown overnight were diluted to an optical density at $600 \mathrm{~nm}\left(\mathrm{OD}_{600}\right)$ of 0.1 in fresh BB10 and then incubated with shaking as described above until an $\mathrm{OD}_{600}$ of 0.12 to 0.15 was reached. The motility of these cultures was confirmed, and they were then used for chemotaxis assays by treating them with L-arginine monohydrochloride (catalog number B577-05; J. T. Baker), sodium fumarate (catalog number 215531000; Acros Organics), L-cysteine hydrochloride monohydrate (catalog number C81020; RPI), D(+)-glucosamine hydrochloride (catalog number 01450; Chem-Impex International Inc.), thiamine hydrochloride (catalog number BP892; Fisher BioReagents), $\alpha$-ketoglutaric acid (catalog number SC-208504; Santa Cruz Biotechnology), or L-malic acid (catalog number 102237; MP Biomedicals) at a final concentration of $0.1 \mathrm{mM}, 1 \mathrm{mM}$, or $10 \mathrm{mM}$ or with an equal volume of $\mathrm{H}_{2} \mathrm{O}$ as a mock-treated control ( $4 \mu \mathrm{l} \mathrm{H}_{2} \mathrm{O}$ or $4 \mu \mathrm{l}$ of a ligand stock in $\mathrm{H}_{2} \mathrm{O}$ into a 96- $\mu$ l culture). The number of direction changes in a bacterial swimming trajectory was enumerated over a 3-s interval to determine whether each putative ligand is sensed as an attractant or repellent or elicits no response (20, 25-30). The results were compared to those with both a repellent control, $10 \mathrm{mM} \mathrm{HCl}$ (catalog number A144S; Fisher Chemical), which results in increased direction changes $(29,31)$, and an attractant control, $50 \mu \mathrm{M}$ 2,2'-dipyridyl (catalog number 117500250; Arcos Organics), which results in fewer direction changes (25). Each control is sensed by chemoreceptors other than TlpA $(24,25,29,31)$. The pH of BB10 upon treatment was independently assessed using a Denver Instruments $\mathrm{pH}$ meter. Prior to realizing that chemotactic responses may be due to medium acidification, we resuspended all ligands in pure water. Therefore, to be able to compare the results to those of previous experiments, we continued resuspending acidified ligands in pure water and then adjusting the $\mathrm{pH}$ of the resuspended ligand using $\mathrm{NaOH}$. Cultures were filmed immediately after ligand addition at a $\times 400$ magnification using a Hamamatsu C4742-95 digital camera with $\mu$ Manager software (version 1.4.22), mounted on a Nikon Eclipse E600 phase-contrast microscope. For the competition chemotaxis assay, cultures of $H$. pylori and ligands were prepared as described above. However, $1 \mathrm{~min}$ after the addition of a nonchemoactive ligand at a final 
concentration of $10 \mathrm{mM}$, a chemoactive ligand was added at a final concentration of $10 \mathrm{mM}$, and the cultures were then filmed as described above. Videos were relabeled to blind the observer to the strain identity. For each sample, $>100$ 3-s-long bacterial tracks from three independent cultures were analyzed manually to identify stops followed by direction changes. Data for all biological replicates under each condition were combined, and the average number of direction changes in $3 \mathrm{~s}$ and the standard error of the mean were calculated. For each strain, data were normalized to the values for the untreated control under each experimental condition. Statistical analysis of the data for treated versus untreated samples was performed using two-way analysis of variance (ANOVA) and Dunnett's multiple-comparison test.

SPR TIpA competition assays. SPR competition assays were performed by using a Biacore S200 instrument and the A-B-A inject function (56). Competition A-B-A analyses were used to interrogate the specificity of the potential ligand binding site preferences of $\operatorname{TlpA}_{\mathrm{LBD}}$ and to unravel the nature of the ligand-sensor interactions. This assay was designed to show if a cumulative response is observed when a second analyte $(B)$ is flown across the bound protein saturated with the first analyte (A) (Fig. 4). As the assay is designed to provide saturation of all analytes tested, this assay does not provide 1:1 competition to indicate which is the preferred analyte for a binding site. The wild-type $T_{1 p A_{L B D}}$ protein was immobilized as described above. A-B-A was used with combinations of each of the compounds (at a concentration 10-fold higher than the equilibrium dissociation constant $\left[K_{D}\right]$ ) and the PBS control, with 60-s injections of analyte A to ensure that saturation or near saturation was reached prior to competition with analyte $B$. The results were analyzed using Biacore S200 evaluation software in the sensorgram mode, and data were zeroed to the baseline before the initial analyte $A$ injection. All response data were normalized to a molecular weight of $100 \mathrm{Da}$ for each analyte, allowing direct comparison of responses. Independent-site theoretical values are calculated by taking the sum of individual responses. Shared-site theoretical values are calculated by taking the sum of individual responses divided by the number of individual responses.

Saturation transfer difference NMR. In the saturation transfer difference (STD) NMR experiment, the entire $\mathrm{TlpA}_{\mathrm{LBD}}$ protein was first saturated at the protein resonances, and excess ligand was then added. As the ligand binds and releases from the receptor, saturation transfers from the protein to the bound ligand. This

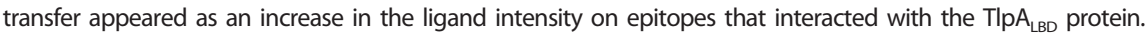
For STD NMR experiments, samples of $25 \mu \mathrm{M}_{\text {TIpA }} \mathrm{LBD}_{\text {in }}$ complex with either $2.5 \mathrm{mM}$ arginine (Arg) or fumaric acid (Fum) in $99 \% \mathrm{D}_{2} \mathrm{O}$ were prepared. All STD NMR spectra were acquired in Shigemi tubes (Shigemi, USA) with a Bruker $600-\mathrm{MHz}$ Advance spectrometer at $283 \mathrm{~K}$ using a $1^{\mathrm{H}}-13^{\mathrm{C}}-15^{\mathrm{N}}$ gradient cryoprobe equipped with z-gradients. Protein resonances were saturated at $-1.0 \mathrm{ppm}$ (on-resonance) and $33 \mathrm{ppm}$ (off-resonance), with a total saturation time of $2 \mathrm{~s}$. A total of 512 scans per STD NMR experiment were acquired, and a Watergate sequence was used to suppress the residual HDO signal. A spin-lock filter with a 5-kHz strength and a duration of $10 \mathrm{~ms}$ was applied to suppress the protein background. On- and off-resonance spectra were stored and processed separately, and the final STD NMR spectra were obtained by subtracting the on- and off-resonance spectra. Control STD NMR experiments were performed identically in the absence of protein.

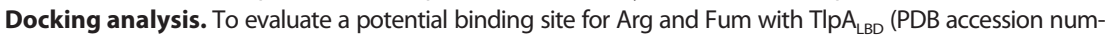
ber 6E09), a blind docking experiment was performed using the AutoDock Vina protocol (60), a high-scoring molecular docking program (32), implemented in the YASARA structure molecular modeling package (version 16.46) (33). The blind docking experiment was set up by using the entire TlpA protein as a potential binding site (grid size, $92.99 \AA \AA$ by $75.73 \AA$ by $62.13 \AA$ ). A total of 999 Vina docking runs were performed.

\section{SUPPLEMENTAL MATERIAL}

Supplemental material is available online only.

FIG S1, PDF file, 0.8 MB.

FIG S2, PDF file, 0.6 MB.

FIG S3, PDF file, 0.6 MB.

FIG S4, PDF file, 0.6 MB.

TABLE S1, PDF file, 1.4 MB.

TABLE S2, PDF file, $0.5 \mathrm{MB}$.

TABLE S3, PDF file, $0.4 \mathrm{MB}$.

TABLE S4, PDF file, $0.4 \mathrm{MB}$.

TABLE S5, PDF file, 0.8 MB.

\section{ACKNOWLEDGMENTS}

We thank Shuai Hu and Xiaolin Liu for their thoughtful comments on this article.

The described project was supported by National Institute of Allergy and Infectious Diseases (NIAID) grant RO1AI116946 to K.M.O. The funders had no role in study design, data collection and interpretation, or the decision to submit the work for publication.

\section{REFERENCES}

1. Matilla MA, Krell T. 2018. The effect of bacterial chemotaxis on host infection and pathogenicity. FEMS Microbiol Rev 42:fux052. https://doi.org/10 .1093/femsre/fux052.
2. Wuichet K, Zhulin IB. 2010. Origins and diversification of a complex signal transduction system in prokaryotes. Sci Signal 3:ra50. https://doi.org/10 $.1126 /$ scisignal.2000724. 
3. Hooi JKY, Lai WY, Ng WK, Suen MMY, Underwood FE, Tanyingoh D, Malfertheiner P, Graham DY, Wong VWS, Wu JCY, Chan FKL, Sung JJY, Kaplan GG, Ng SC. 2017. Global prevalence of Helicobacter pylori infection: systematic review and meta-analysis. Gastroenterology 153:420-429. https://doi.org/10.1053/j.gastro.2017.04.022.

4. Cover TL, Blaser MJ. 2009. Helicobacter pylori in health and disease. Gastroenterology 136:1863-1873. https://doi.org/10.1053/j.gastro.2009.01 .073 .

5. Plummer M, Franceschi S, Vignat J, Forman D, de Martel C. 2015. Global burden of gastric cancer attributable to Helicobacter pylori. Int J Cancer 136:487-490. https://doi.org/10.1002/ijc.28999.

6. Ferlay J, Soerjomataram I, Dikshit R, Eser S, Mathers C, Rebelo M, Parkin DM, Forman D, Bray F. 2015. Cancer incidence and mortality worldwide: sources, methods and major patterns in GLOBOCAN 2012. Int J Cancer 136:E359-E386. https://doi.org/10.1002/ijc.29210.

7. Wroblewski LE, Peek RM, Wilson KT. 2010. Helicobacter pylori and gastric cancer: factors that modulate disease risk. Clin Microbiol Rev 23:713-739. https://doi.org/10.1128/CMR.00011-10.

8. White JR, Winter JA, Robinson K. 2015. Differential inflammatory response to Helicobacter pylori infection: etiology and clinical outcomes. J Inflamm Res 8:137-147. https://doi.org/10.2147/JIR.S64888.

9. Arnold IC, Lee JY, Amieva MR, Roers A, Flavell RA, Sparwasser T, Müller A. 2011. Tolerance rather than immunity protects from Helicobacter pylori induced gastric preneoplasia. Gastroenterology 140:199-209. https://doi .org/10.1053/j.gastro.2010.06.047.

10. Blosse A, Lehours P, Wilson KT, Gobert AP. 2018. Helicobacter: inflammation, immunology, and vaccines. Helicobacter 23:e12517. https://doi.org/ 10.1111/hel.12517.

11. Javed S, Skoog EC, Solnick JV. 2019. Impact of Helicobacter pylori virulence factors on the host immune response and gastric pathology. Curr Top Microbiol Immunol 421:21-52. https://doi.org/10.1007/978-3-030 -15138-6_2.

12. Johnson KS, Ottemann KM. 2018. Colonization, localization, and inflammation: the roles of $H$. pylori chemotaxis in vivo. Curr Opin Microbiol 41:51-57. https://doi.org/10.1016/j.mib.2017.11.019.

13. McGee DJ, Langford ML, Watson EL, Carter JE, Chen Y-T, Ottemann KM. 2005. Colonization and inflammation deficiencies in Mongolian gerbils infected by Helicobacter pylori chemotaxis mutants. Infect Immun 73:1820-1827. https://doi.org/10.1128/IAI.73.3.1820-1827.2005.

14. Rolig AS, Carter JE, Ottemann KM. 2011. Bacterial chemotaxis modulates host cell apoptosis to establish a T-helper cell, type 17 (Th17)-dominant immune response in Helicobacter pylori infection. Proc Natl Acad Sci U S A 108:19749-19754. https://doi.org/10.1073/pnas.1104598108.

15. Williams SM, Chen Y-T, Andermann TM, Carter JE, McGee DJ, Ottemann KM. 2007. Helicobacter pylori chemotaxis modulates inflammation and bacterium-gastric epithelium interactions in infected mice. Infect Immun 75:3747-3757. https://doi.org/10.1128/IAI.00082-07.

16. Andermann TM, Chen Y-T, Ottemann KM. 2002. Two predicted chemoreceptors of Helicobacter pylori promote stomach infection. Infect Immun 70:5877-5881. https://doi.org/10.1128/IAI.70.10.5877-5881.2002.

17. Rolig AS, Shanks J, Carter JE, Ottemann KM. 2012. Helicobacter pylori requires TIpD-driven chemotaxis to proliferate in the antrum. Infect Immun 80:3713-3720. https://doi.org/10.1128/IAI.00407-12.

18. Upadhyay AA, Fleetwood AD, Adebali O, Finn RD, Zhulin IB. 2016. Cache domains that are homologous to, but different from PAS domains comprise the largest superfamily of extracellular sensors in prokaryotes. PLoS Comput Biol 12:e1004862. https://doi.org/10.1371/journal.pcbi.1004862.

19. Sweeney EG, Perkins A, Kallio K, Remington SJ, Guillemin K. 2018. Structures of the ligand-binding domain of Helicobacter pylori chemoreceptor TIpA. Protein Sci 27:1961-1968. https://doi.org/10.1002/pro.3503.

20. Machuca MA, Johnson KS, Liu YC, Steer DL, Ottemann KM, Roujeinikova A. 2017. Helicobacter pylori chemoreceptor TIpC mediates chemotaxis to lactate. Sci Rep 7:14089. https://doi.org/10.1038/s41598-017-14372-2.

21. McKellar JLO, Minnell JJ, Gerth ML. 2015. A high-throughput screen for ligand binding reveals the specificities of three amino acid chemoreceptors from Pseudomonas syringae pv. actinidiae. Mol Microbiol 96:694-707. https://doi.org/10.1111/mmi.12964.

22. Cerda O, Rivas A, Toledo H. 2003. Helicobacter pylori strain ATCC700392 encodes a methyl-accepting chemotaxis receptor protein (MCP) for arginine and sodium bicarbonate. FEMS Microbiol Lett 224:175-181. https:// doi.org/10.1016/S0378-1097(03)00423-3.

23. Cerda OA, Núñez-Villena F, Soto SE, Ugalde JM, López-Solís R, Toledo H. 2011. t/pA gene expression is required for arginine and bicarbonate chemotaxis in Helicobacter pylori. Biol Res 44:277-282. https://doi.org/10 .4067/S0716-97602011000300009.

24. Huang JY, Goers Sweeney E, Guillemin K, Amieva MR. 2017. Multiple acid sensors control Helicobacter pylori colonization of the stomach. PLoS Pathog 13:e1006118. https://doi.org/10.1371/journal.ppat.1006118.

25. Collins KD, Andermann TM, Draper J, Sanders L, Williams SM, Araghi C, Ottemann KM. 2016. The Helicobacter pylori CZB cytoplasmic chemoreceptor TIpD forms an autonomous polar chemotaxis signaling complex that mediates a tactic response to oxidative stress. J Bacteriol 198:1563-1575. https://doi.org/10.1128/JB.00071-16.

26. Terry K, Go AC, Ottemann KM. 2006. Proteomic mapping of a suppressor of non-chemotactic cheW mutants reveals that Helicobacter pylori contains a new chemotaxis protein. Mol Microbiol 61:871-882. https://doi .org/10.1111/j.1365-2958.2006.05283.x.

27. Lertsethtakarn P, Howitt MR, Castellon J, Amieva MR, Ottemann KM. 2015. Helicobacter pylori CheZHP and ChePep form a novel chemotaxis-regulatory complex distinct from the core chemotaxis signaling proteins and the flagellar motor: $H$. pylori CheZ HP and ChePep form a distinct complex. Mol Microbiol 97:1063-1078. https://doi.org/10.1111/mmi.13086.

28. Schweinitzer T, Mizote T, Ishikawa N, Dudnik A, Inatsu S, Schreiber S, Suerbaum S, Aizawa S-I, Josenhans C. 2008. Functional characterization and mutagenesis of the proposed behavioral sensor TIpD of Helicobacter pylori. J Bacteriol 190:3244-3255. https://doi.org/10.1128/JB.01940-07.

29. Goers Sweeney E, Henderson JN, Goers J, Wreden C, Hicks KG, Foster JK, Parthasarathy R, Remington SJ, Guillemin K. 2012. Structure and proposed mechanism for the $\mathrm{pH}$-sensing Helicobacter pylori chemoreceptor TlpB. Structure 20:1177-1188. https://doi.org/10.1016/j.str.2012.04.021.

30. Rader BA, Wreden C, Hicks KG, Sweeney EG, Ottemann KM, Guillemin K. 2011. Helicobacter pylori perceives the quorum-sensing molecule Al-2 as a chemorepellent via the chemoreceptor TlpB. Microbiology (Reading) 157:2445-2455. https://doi.org/10.1099/mic.0.049353-0.

31. Croxen MA, Sisson G, Melano R, Hoffman PS. 2006. The Helicobacter pylori chemotaxis receptor TlpB (HP0103) is required for $\mathrm{pH}$ taxis and for colonization of the gastric mucosa. J Bacteriol 188:2656-2665. https://doi.org/ 10.1128/JB.188.7.2656-2665.2006.

32. Wang Z, Sun H, Yao X, Li D, Xu L, Li Y, Tian S, Hou T. 2016. Comprehensive evaluation of ten docking programs on a diverse set of protein-ligand complexes: the prediction accuracy of sampling power and scoring power. Phys Chem Chem Phys 18:12964-12975. https://doi.org/10.1039/ c6cp01555g.

33. Krieger E, Koraimann G, Vriend G. 2002. Increasing the precision of comparative models with YASARA NOVA-a self-parameterizing force field. Proteins 47:393-402. https://doi.org/10.1002/prot.10104.

34. Biemann HP, Koshland DE. 1994. Aspartate receptors of Escherichia coli and Salmonella typhimurium bind ligand with negative and half-of-thesites cooperativity. Biochemistry 33:629-634. https://doi.org/10.1021/ bi00169a002.

35. Haselhorst T, Lamerz A-C, von Itzstein M. 2009. Saturation transfer difference NMR spectroscopy as a technique to investigate protein-carbohydrate interactions in solution, p 375-396. In Packer NH, Karlsson NG (ed), Glycomics. Humana Press, Totowa, NJ.

36. Mayer M, Meyer B. 1999. Characterization of ligand binding by saturation transfer difference NMR spectroscopy. Angew Chem Int Ed Engl 38:1784-1788. https://doi.org/10.1002/(SICI)1521-3773(19990614)38: $12<1784:$ :AID-ANIE1784>3.0.CO;2-Q.

37. Martín-Mora D, Ortega Á, Pérez-Maldonado FJ, Krell T, Matilla MA. 2018. The activity of the C4-dicarboxylic acid chemoreceptor of Pseudomonas aeruginosa is controlled by chemoattractants and antagonists. Sci Rep 8:2102. https://doi.org/10.1038/s41598-018-20283-7.

38. Bi S, Yu D, Si G, Luo C, Li T, Ouyang Q, Jakovljevic V, Sourjik V, Tu Y, Lai L. 2013. Discovery of novel chemoeffectors and rational design of Escherichia coli chemoreceptor specificity. Proc Natl Acad Sci U S A 110:16814-16819. https://doi.org/10.1073/pnas.1306811110.

39. Rahman H, King RM, Shewell LK, Semchenko EA, Hartley-Tassell LE, Wilson JC, Day CJ, Korolik V. 2014. Characterisation of a multi-ligand binding chemoreceptor CcmL (Tlp3) of Campylobacter jejuni. PLoS Pathog 10: e1003822. https://doi.org/10.1371/journal.ppat.1003822.

40. Ud-Din AIMS, Khan MF, Roujeinikova A. 2020. Broad specificity of amino acid chemoreceptor CtaA of Pseudomonas fluorescens is afforded by plasticity of its amphipathic ligand-binding pocket. Mol Plant Microbe Interact 33:612-623. https://doi.org/10.1094/MPMI-10-19-0277-R.

41. Nedenskov P. 1994. Nutritional requirements for growth of Helicobacter pylori. Appl Environ Microbiol 60:3450-3453. https://doi.org/10.1128/aem .60.9.3450-3453.1994. 
42. McGee DJ, Radcliff FJ, Mendz GL, Ferrero RL, Mobley HLT. 1999. Helicobacter pylori rocF is required for arginase activity and acid protection in vitro but is not essential for colonization of mice or for urease activity. J Bacteriol 181:7314-7322. https://doi.org/10.1128/JB.181.23.7314-7322.1999.

43. Gobert AP, Wilson KT. 2016. The immune battle against Helicobacter pylori infection: NO offense. Trends Microbiol 24:366-376. https://doi.org/10 .1016/j.tim.2016.02.005.

44. Alam M, Srivastava A, Dutta A, Sau AK. 2018. Biochemical and biophysical studies of Helicobacter pylori arginine decarboxylase, an enzyme important for acid adaptation in host. IUBMB Life 70:658-669. https://doi.org/ 10.1002/iub.1754.

45. Valenzuela M, Cáceres A, Almarza O, Bravo D, Soto S, Cerda O, Toledo H. 2014. Characterization of the arginine decarboxylase gene (ORF HP0422, speA) involved in acid tolerance in Helicobacter pylori. Helicobacter 19:182-193. https://doi.org/10.1111/hel.12115.

46. Mendz GL, Hazell SL, Srinivasan S. 1995. Fumarate reductase: a target for therapeutic intervention against Helicobacter pylori. Arch Biochem Biophys 321:153-159. https://doi.org/10.1006/abbi.1995.1380.

47. Ge Z, Feng Y, Dangler CA, Xu S, Taylor NS, Fox JG. 2000. Fumarate reductase is essential for Helicobacter pylori colonization of the mouse stomach. Microb Pathog 29:279-287. https://doi.org/10.1006/mpat.2000.0391.

48. Keilberg D, Steele N, Fan S, Yang C, Zavros Y, Ottemann KM. 2021. Gastric metabolomics detects Helicobacter pylori correlated loss of numerous metabolites in both the corpus and antrum. Infect Immun 89:e00690-20. https://doi.org/10.1128/IAI.00690-20.

49. Nishiumi S, Yoshida M, Azuma T. 2017. Alterations in metabolic pathways in stomach of mice infected with Helicobacter pylori. Microb Pathog 109:78-85. https://doi.org/10.1016/j.micpath.2017.05.027.

50. Busch A, Lacal J, Martos A, Ramos JL, Krell T. 2007. Bacterial sensor kinase TodS interacts with agonistic and antagonistic signals. Proc Natl Acad Sci U S A 104:13774-13779. https://doi.org/10.1073/pnas.0701547104.

51. Hartley-Tassell LE, Shewell LK, Day CJ, Wilson JC, Sandhu R, Ketley JM, Korolik V. 2010. Identification and characterization of the aspartate chemosensory receptor of Campylobacter jejuni: the aspartate receptor of Campylobacter jejuni. Mol Microbiol 75:710-730. https://doi.org/10.1111/j .1365-2958.2009.07010.x.
52. Krell T. 2015. Tackling the bottleneck in bacterial signal transduction research: high-throughput identification of signal molecules. Mol Microbiol 96:685-688. https://doi.org/10.1111/mmi.12975.

53. Ehrhardt MKG, Warring SL, Gerth ML. 2018. Screening chemoreceptorligand interactions by high-throughput thermal-shift assays. Methods Mol Biol 1729:281-290. https://doi.org/10.1007/978-1-4939-7577-8_22.

54. Lee WC, Goh KL, Loke MF, Vadivelu J. 2017. Elucidation of the metabolic network of Helicobacter pylori $\mathrm{J} 99$ and Malaysian clinical strains by phenotype microarray. Helicobacter 22:e12321. https://doi.org/10.1111/hel.12321.

55. Lertsethtakarn P, Ottemann KM, Hendrixson DR. 2011. Motility and chemotaxis in Campylobacter and Helicobacter. Annu Rev Microbiol 65:389-410. https://doi.org/10.1146/annurev-micro-090110-102908.

56. Elgamoudi BA, Andrianova EP, Shewell LK, Day CJ, King RM, Taha, Rahman H, Hartley-Tassell LE, Zhulin IB, Korolik V. 2021. The Campylobacter jejuni chemoreceptor Tlp10 has a bimodal ligand-binding domain and specificity for multiple classes of chemoeffectors. Sci Signal 14: eabc8521. https://doi.org/10.1126/scisignal.abc8521.

57. Day CJ, Korolik V. 2018. Identification of specific ligands for sensory receptors by small-molecule ligand arrays and surface plasmon resonance. Methods Mol Biol 1729:303-317. https://doi.org/10.1007/978-1-4939-7577-8 24.

58. Liu Y, McBride R, Stoll M, Palma AS, Silva L, Agravat S, Aoki-Kinoshita KF, Campbell MP, Costello CE, Dell A, Haslam SM, Karlsson NG, Khoo K-H, Kolarich D, Novotny MV, Packer NH, Ranzinger R, Rapp E, Rudd PM, Struwe WB, Tiemeyer M, Wells L, York WS, Zaia J, Kettner C, Paulson JC, Feizi T, Smith DF. 2017. The minimum information required for a glycomics experiment (MIRAGE) project: improving the standards for reporting glycan microarray-based data. Glycobiology 27:280-284. https://doi.org/ 10.1093/glycob/cww118.

59. Day CJ, King RM, Shewell LK, Tram G, Najnin T, Hartley-Tassell LE, Wilson JC, Fleetwood AD, Zhulin IB, Korolik V. 2016. A direct-sensing galactose chemoreceptor recently evolved in invasive strains of Campylobacter jejuni. Nat Commun 7:13206. https://doi.org/10.1038/ncomms13206.

60. Trott O, Olson AJ. 2010. AutoDock Vina: improving the speed and accuracy of docking with a new scoring function, efficient optimization, and multithreading. J Comput Chem 31:455-461. https://doi.org/10.1002/jcc .21334 . 